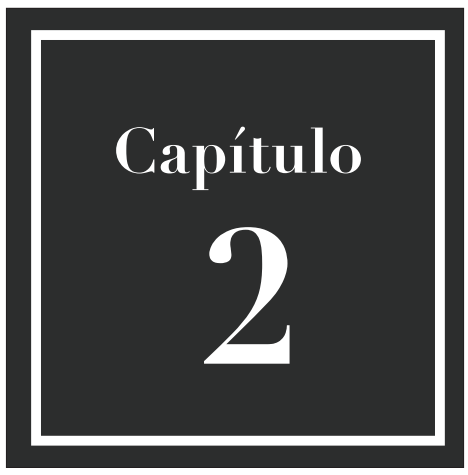

\title{
EN ALERTA LA COMUNIDAD CIENTÍFICA AMBIENTAL INTERNACIONAL
}

\author{
Un poco de historia
}

El panorama estremecedor desglosado en el capítulo anterior ha puesto en alerta a la comunidad científica ambiental internacional que, a pesar de existir el Convenio Ramsar, firmado en Irán en 1971, siendo su principal objetivo "la conservación y el uso racional de los humedales mediante acciones locales, regionales y nacionales y gracias a la cooperación internacional, como contribución al logro de un desarrollo sostenible en todo el mundo", en su lista existen al menos dos mil humedales en 160 países, los cuales poseen planes de uso racional para su protección, debido a que presentan alta diversidad biológicva, además de ser reguladores del clima y controlar las inundaciones, suministrar agua a las poblaciones aledañas y tener gran potencial turístico y recreacional, pero siguen siendo muy pocos los países que acatan este convenio.

Hoy en día, al menos la mitad de la población mundial está asentada en zonas urbanas; se estima aproximadamente cuatro mil millones de personas, lo que representa una peligrosa amenaza en la ocupación de los humedales y, por ende, para su protección, uso y manejo. Estos humedales urbanos aportan beneficios económicos, sociales y culturales a las ciudades cuando se utilizan de forma sostenible, pues- to que, durante las tormentas, los humedales absorben el exceso de lluvia, reduciendo las inundaciones en las ciudades y evitando catástrofes, con los costos que estas conllevan. Así mismo, la abundante vegetación que poseen actúa como filtro de residuos domésticos e industriales y contribuye a una mejor calidad del agua y, por supuesto, a una mejor calidad de vida de sus habitantes.

La mencionada Convención Ramsar ha reconocido 18 ciudades en el mundo que han tomado medidas de conservación de manera progresiva hacia el urbanismo sostenible; estas son: Changde, Changshu, Dongying, Haerbin, Haikou y Yinchuan, en China; Amiens, Courteranges, Pont-Audemer y Saint Omer, en Francia; Lakes by Tata, en Hungría, Changnyeong, Inje, Jeju y Suncheon, en República de Corea; Mitsinjo, en Madagascar; Colombo, en Sri Lanka y Ghar el Melh, en Túnez. Esta acreditación de ciudad de humedal de importancia internacional da cabida, mediante la planificación, a tomar conciencia en la población del uso y manejo de estos ecosistemas urbanos.

En América Latina, numerosos son los sitios de humedales que deberían optar por la cate- 
goría Ramsar, pero la realidad es otra, quizás por la falta de interés político que existe para la conservación de estos ecosistemas. Sin embargo, Colombia ha logrado convertirse en el único país de Latinoamérica con un complejo de humedales urbanos dentro de esa categoría de protección internacional. Se destaca su capital, Bogotá, por poseer once humedales urbanos que representan 667,38 ha. Estos son: Tibanica, La Vaca Norte, El Burro, El Tunjo, Capellanía, Santa María del Lago, Córdoba, Jaboque, Juan Amarillo, La Conejera y Torca-Guaymaral, que son puntos estratégicos de aves migratorias para el descanso y alimentación, así como para otras especies vegetales, mamíferos y aves habituales y acuáticas. Lo mencionado se traduce en beneficios ecosistémicos que proporcionan los humedales urbanos para una población total de 7.181.469 habitantes en la capital, según las cifras del Censo de Población y Vivienda del Departamento Administrativo Nacional de Estadística, DANE (2018).

En otro orden de ideas, existe un sinnúmero de estudios de especialistas dedicados a la investigación, uso y manejo de los humedales; sin embargo, son pocos sobre humedales urbanos específicamente. Entre los más destacados se puede nombrar el de Rojas (2015): "Cambios ambientales y dinámica de inundaciones fluviales en una cuenca costera del centro sur de Chile", el cual indica que la urbanización en las últimas décadas ha impactado áreas de valor ecológico, donde incluye a los humedales. Analiza los cambios, la dinámica y la perturbación de parámetros de la biodiversidad en humedales urbanos, por medio de la exploración y uso de indicadores.

Según los resultados del estudio, los humedales con menor grado de perturbación antrópica se encuentran ubicados en una reserva protegida y rodeados de paisaje seminatural; mientras que los más alterados están más cercanos a la urbanización. Además, las especies se ven más afectadas por la distancia a carreteras, y la riqueza de plantas por la densidad de viviendas. Por lo tanto, se crea un instrumento para la valoración de efectos de la urbanización en los humedales. Por otro lado, se comprueban las tendencias de aumento en la precipitación extrema y el acrecentamiento de la urbanización, dando como resultado un mayor riesgo a las inundaciones, por lo tanto, debe haber una ocupación del territorio más sustentable y menos sensible a este tipo de desastres.

Igualmente, Valencia y Figueroa (2014), en "Vulnerabilidad de humedales alto andinos ante procesos de cambio: tendencias del análisis", que buscó hacer una revisión de algunos referentes de los procesos de degradación de los humedales altoandinos de Colombia, exponiendo las tendencias de los análisis de la vulnerabilidad y resaltando el reto actual de desarrollar los estudios por medio de una visión que integre lo social y lo biofísico, a partir de un enfoque sistémico de estos sistemas naturales, para introducir la visión de los sistemas socio-ecológicos o socio-ecosistemas como una opción ya conocida, pero que debe ser desarrollada y apropiada para su implantación y comprensión por las comunidades.

Estos autores hicieron posible entender el estado y la vulnerabilidad ante los procesos de intervención, deterioro y conservación, al dejar de ver los cuerpos de agua como espacios intocables para verlos como un sistema dinámico que une al ser humano en la naturaleza. Demostraron, utilizando una metodología bajo un enfoque cuantitativo, a través de los modelos: medios de vida sostenibles, de Chambers y Conway (1992), presión-liberación, de Blaikie et al. (1994), doble estructura, de Bohle et al., (1994), de lugar, de Cutter (1996), riesgo-amenaza, de Davidson (1997) y desde cambio global, de Turner (2003), que la vulnerabilidad debe analizarse como una posición integral, multifactorial, multisectorial, multitemporal y multiescalar. Lo expuesto permite relacionar los sistemas sociales con los ecosistemas, integrando de una manera global las interacciones entre ellos para el mejor conocimiento de la vulnerabilidad de los humedales frente a la intervención antrópica.

En cuanto a planes de manejo existen innumerables trabajos a nivel internacional para los humedales, no así en Venezuela, por lo tanto, se tomó como referencia el trabajo realizado por la Alcaldía de Bogotá, Colombia (2006), titulado "Plan de manejo humedal La Vaca", siendo uno de los más aplicados a la problemática que presentan los humedales urbanos.

En Venezuela existe la majestuosa obra de Marrero (2018), "Introducción a los humedales altoandinos de Venezuela", la cual constituye una importante contribución al conocimiento y comprensión de los humedales altoandinos venezolanos como espacios naturales que evolucionan en su interacción con los grupos humanos. Este trabajo recoge muchas de esas investigaciones y las relaciona con la compleja realidad social y económica de estos ecosistemas. Su valor didáctico permite conocer la diversidad que existe en los pisos altitudinales del paisaje andino, su biodiversidad 
más representativa y los problemas derivados de las complejas interacciones entre los seres humanos con estos.

Así, este estudio plantea la situación de los humedales, la problemática, así como también las amenazas a las que están expuestos estos ecosistemas en Venezuela. También define y caracteriza los humedales altoandinos venezolanos, los factores físicos que los determinan, su ubicación y clasificación, su formación y funcionamiento y amenazas.

Otra investigación relevante, es la de Ayube (2012), "Los humedales en el sistema legal venezolano", donde planteó que no existe en Venezuela ningún estudio de profundidad sobre el sustrato teórico y legal de los humedales que brinde una situación real de su estado actual, de la problemática que presentan y de las posibilidades de manejo sustentable. Se realizó por la necesidad de estudiar y analizar todos los aspectos de los humedales a fin de hacerlos ocupar el lugar que realmente tienen. Además, resaltó que estos conocimientos son necesarios para su administración y protección.

Este trabajo es de tipo documental, puesto que la autora, recopiló y analizó un volumen de información, instrumentos y pautas, con la finalidad de presentar los fundamentos teóricos que de una $u$ otra forma se relacionan los humedales, su manejo, clasificación y problemas ambientales que afrontan en la actualidad como áreas naturales protegidas dentro del marco jurídico-ambiental. Concluye la autora que, tomando en consideración que el uso del territorio debe estar normado y debe tener un sustento legal para su administración, así se asegura el manejo adecuado de los humedales urbanos.

Por su parte, Yépez (2010), en su tesis doctoral "Patrones ecogeohídricos en un sistema de humedales del páramo Mifafí (nacientes del río Chama), cordillera de Mérida-Venezuela", sostiene que:

Actualmente los humedales están siendo intervenidos por actividades antrópicas, en particular el pastoreo extensivo de bovinos y equinos, así como la agricultura y el turismo rural, generando cambios o efectos adversos que desencadenan conflictos de uso por los recursos agua, suelo y vegetación. Además de las amenazas por intervención antrópica se evidencian amenazas asociadas a los cambios climáticos, lo que redundará en una intensificación del ciclo hidrológico y podrían tener importantes impactos en los recursos hídricos regionales.

Igualmente, el conocimiento científico sobre el funcionamiento de los humedales podría influir significativamente en la toma de decisiones con respecto al uso actual y futuro de los espacios donde se localizan estos ecosistemas. El objetivo de esta investigación fue definir y caracterizar patrones ecogeohídricos, en la cuenca de la quebrada Mifafí, sobre la base del papel que estos patrones desempeñan en la formación de los humedales de páramo y su función hídrica como almacenadores o reservorios. (Yépez, 2010, p.75)

La autora siguió una metodología bajo enfoque cuantitativo, se centró en tres fases. Primera: compilación de información topográfica, climática, edafológica, hidrométrica y de uso del suelo a nivel de cuenca hidrográfica. Medida de variables en periodo seco y lluvioso. Segunda: elección del complejo de humedales. Cálculo de variables en periodo seco y lluvioso. Tercera: caracterización de los patrones ecogeohídricos y clarificación preliminar de humedales altoandinos.

Asimismo, clasificó los humedales a nivel de sistemas en fluviales, depresionales y de ladera e identificó subsistemas en: ciénagas en laderas de conos o abanicos, ciénagas en contacto con el fondo de valle-ladera, céspedes en fondo de valle, céspedes de ladera en conos coalescentes, céspedes en la base de acumulaciones morrenicas y lagunas glaciales. Los humedales del páramo de Mifafí cumplen funciones de acumuladores de agua durante todo el año. Asegura la autora que de la ordenación del territorio depende la duración de estos ecosistemas, mermando a la vez las consecuencias de la agricultura y el turismo. Esta investigación comprueba que de la adecuación y manejo de los humedales depende la producción de agua, en cantidad y calidad, en las cuencas hidrográficas, lo que asegura un buen servicio de abastecimiento a las comunidades (Yépez, 2010).

\section{U N A S E R E D E C O N G E C I O N E S}

Seguidamente se muestran los referentes teóricos, un poco para entender los términos utilizados en el tema presentado. Así, Saunier (1987) define como manejo ambiental: "El conjunto detallado de actividades, que producto de una evaluación ambiental, están orientadas a prevenir, mitigar, corregir o compensar los im- 
pactos y efectos ambientales que se causen por el desarrollo de un proyecto, obra o actividad. Incluye los planes de seguimiento, monitoreo, contingencia, y abandono según la naturaleza del proyecto, obra o actividad" (p.2). Se refiere a las acciones que se requieren para los posibles efectos o impactos ambientales negativos causados en el desarrollo de actividades humanas. Estas acciones son conducidas por los organismos de competencia gubernamental aunada con la cooperación de la comunidad. Es decir, la gestión gobierno, comunidad y ambiente (Saunier, 1987).

Así que el manejo ambiental basado en los fundamentos ecológicos esgrime el análisis y métodos de sistema y solución de conflictos para distribuir los costos y beneficios de las actividades de desarrollo en las poblaciones afectadas, y procura protegerlas de los riesgos naturales. Lo cual constituye un desarrollo ambientalmente apropiado. En la actualidad, el manejo ambiental es fundamental porque las actividades de desarrollo que se llevan a cabo en un sector afectan en forma positiva y/o negativa en la calidad de la vida de otras comunidades, es lo que se designa como impacto ambiental.

En cuanto al acondicionamiento ambiental, se debe tomar en cuenta que el hombre al asentarse en un lugar se encuentra condicionado por las características naturales, llamado medio físico natural; este proceso produce, al efectuar las modificaciones en su acomodo al sitio electo para el asentamiento, una interrelación inmediata con los factores del medio, con mayor o menor roce según se precisen los contextos de acomodamiento. Así que cuanto más se observen las formas de adecuación del medio físico transformado, el asentamiento construido, en el medio natural y menor sea el impacto sobre este, se garantizará la calidad del asentamiento y su desarrollo sostenible.

Por lo expuesto, el acondicionamiento ambiental está referido al estudio de las transformaciones de los elementos de instalaciones y el urbanismo que tiene sobre el clima a la preparación del exterior. Es la adecuación de lo construido por el ser humano en conformidad con el medio circundante, alterando lo menos posible el ambiente (Vásquez, 2012). De acuerdo con esto, el mismo autor lo conceptualiza como "el estudio de las modificaciones de los elementos de la arquitectura y del urbanismo que tienen sobre los elementos del clima total al medio exterior" (Vásquez, 2012, p.1).
En cuanto a los factores externos que influyen en el acondicionamiento ambiental, Schjetnan (2012), hace referencia a la temperatura, los vientos, la humedad, la radiación solar, la vegetación, la acústica, entre otros, con el fin de encontrar un confort del ambiente y lograr un excelente diseño urbano eficiente buscando la manera de aprovechar las condiciones climáticas favorables y de ajustar las condiciones desfavorables; es decir, el acondicionamiento del espacio físico mejorando las condiciones ambientales y urbanas que faciliten el desarrollo interno y permita su combinación con el resto de la ciudad.

Así, el acondicionamiento ambiental es un proceso de adecuación del espacio a un proceso de desarrollo integral, orientado a estructuración de infraestructura y usos de suelo en función del eficiente proceso productivo y de calidad de hábitat de población. Este proceso de adecuación, según Schjetnan (2012), está orientado a la adecuación al clima,

\begin{abstract}
a la temperatura como componente del medio natural, es la cantidad de calor que existe en la atmósfera, procedente del sol; está en función de diversos factores, como: a) inclinación de incidencia de los rayos solares sobre la superficie que tiene una variación según hora del día, época del año y distancia a línea del Ecuador o ubicación geográfica. b) el reflejo que producen los rayos solares, tanto en la superficie terrestre como en las aguas, de acuerdo con la función de absorción que tiene. Las superficies de agua tienen una inercia mayor que las masas de aire, las superficies de agua templan el aire caliente y muy frío. (p.14)
\end{abstract}

El efecto global de temperatura - aire - sol, se deriva principalmente del comportamiento de la superficie expuesta a las radiaciones, las cubiertas de vegetación y las aguas que absorben lentamente y lo pierden en más tiempo, no así la tierra que muestra alta permeabilidad, pero la pérdida es más rápida por la noche. La temperatura del aire tiene una consecuencia térmica en los interiores a causa de la filtración del aire. El control de la temperatura en las construcciones es determinante para el diseño urbano, en el que se debe considerar la utilización de vegetación y de agua, según el clima del lugar, como elementos refrescantes o de protección. Igualmente, una reglamentación adecuada para las construcciones puede favorecer al control de la temperatura, con el consiguiente ahorro del uso de ventiladores, calefactores o de aire acondicionado.

Por otra parte, la adecuación a las masas de aire ocasionados por cambios de presión at- 
mosférica, pueden ser: a) Regulares: tienen una misma dirección durante todo el año. b) Periódicos: cambios de dirección cada determinado tiempo, por un período largo o, como en la montaña y el valle, donde el viento asciende a las partes más altas durante el día y por las noches desciende. c) Irregulares: ciclones, tornados y las trombas. El movimiento del aire en un espacio tiene como función, además de activar la renovación del aire, acelerar el proceso de conversión térmica.

En cuanto a la adecuación a las precipitaciones, su origen se debe al enfriamiento del aire, que hace que el vapor de agua contenido en las nubes se convierta en gotas de agua y se precipiten en forma de lluvia. De acuerdo con la cantidad de precipitaciones y la duración de las épocas lluviosas, el diseño urbano debe prever y dimensionar los sistemas de drenaje y escurrimiento, así como los tipos de pavimentos. Las características de asentamientos en zonas cálidas dependen de la necesidad de protección de las lluvias. Por otra parte, las construcciones, según el material predominante en la región, deben elaborarse con cubiertas inclinadas, como sea la cantidad y periodicidad de las lluvias y adecuarse a las redes de drenaje.

Simultáneamente, la humedad es considerada como la cantidad de vapor de agua en las partes bajas de la atmósfera, resultado de la evaporación de las masas de agua, terrenos húmedos y la transpiración de las plantas. Por el hecho de tener una inercia térmica mayor que las masas de aire, las superficies de agua moderan el aire muy caliente y el muy frío, teniendo en cuenta que los ríos mantienen el clima fresco y húmedo y la evaporación del agua aumenta la humedad.

En climas muy cálidos, con evaporación acentuada, se tiene una favorable disminución de la acción directa de los rayos solares. El índice de nubosidad y la transparencia atmosférica, que tienen influencia sobre las radiaciones solares, están relacionados con el contenido de agua atmosférica. De modo que la humedad ambiental es importante para las tipologías constructivas, sobre todo en lo que se refiere a la selección adecuada de materiales de construcción duraderos y resistentes a la acción de la humedad para acabados, aislantes y revestimientos de obras y de edificaciones.

En otro orden de ideas, los humedales urbanos surgen como un nuevo paradigma en el contexto de la ecología y conservación de humedales y su relación con el desarrollo de las grandes ciudades. Son humedales urbanos porque han quedado inmersos en la ciudad, en la matriz urbana, rodeándose de gente, su cultura y de sus necesidades; su entorno ha sido transformado por el crecimiento incontrolado de las ciudades. Su funcionamiento en buena medida es controlado por el aprovechamiento de la ciudad y el movimiento de sus aguas (Ramsar, 2016). En cuanto a la importancia de los humedales urbanos está en su contribución a la diversidad biológica y belleza del paisaje. Según Smith y Romero (2009):

\section{Los humedales ubicados dentro de la ciudad o cer- canos a ella, adquieren aún mayor importancia debido a que las funciones y mecanismos natura- les de estos ecosistemas deben ser entendidos como servicios ambientales que reportan beneficios di- rectos e indirectos a la humanidad, entre los que se encuentran purificación de aire, regulación mi- croclimática, reducción de ruido, drenaje de aguas lluvias, tratamiento de aguas residuales y oferta de espacios para la recreación. (p.81)}

De aquí se desprende la fundamentación en la decisión de conservar estos ecosistemas dentro de una ciudad. Es de destacar que el ordenamiento territorial es una política de Estado y un instrumento de planificación. Como política de Estado debe garantizar una apropiada organización política administrativa, la proyección espacial de las políticas de desarrollo social, económico, ambiental y cultural, con el objetivo de alcanzar un nivel de vida apropiado para la población asentada en estos ecosistemas y, a su vez, que permita la conservación del ambiente dentro de las políticas del desarrollo sostenible. Estas políticas se traducen en ordenación del territorio como un conjunto de administraciones públicas y planificaciones encaminadas a la coordinación y compatibilización de los usos y funciones que coexisten en un determinado territorio; es decir, el conjunto de disposiciones y funciones legales, planes, programas y actuaciones que, de manera integrada, desarrolla un gobierno de modo que las actuaciones territoriales respondan a los deseos de bienestar y, en general, a mejorar y elevar la calidad de vida de las personas, individual y socialmente.

Por lo tanto, el ordenamiento territorial, orienta la ocupación y utilización adecuada de los diversos espacios geográficos, en forma equilibrada y sustentable, considerando sus potenciales, limitantes y problemática existente. No se debe dejar de constatar que existen lineamientos adecuados para las prescripciones de la Convención sobre la conservación de los humedales inscritos en la lista de humedales de importancia internacional y el uso racional de 
los humedales situados en el territorio de las partes contratantes, tal como lo cita el artículo $\mathrm{N}^{\mathrm{o}} 3$ de dicha Convención, así como para el establecimiento de reservas naturales (áreas protegidas) en humedales, que figuren o no en la lista Ramsar.

Efectivamente, si se cuenta con acuerdos administrativos y legales que responden a la protección, al manejo de cada ecosistema, así como la existencia de las bases legales adecuadas, las cuales son elementos fundamentales para el éxito a largo plazo de estos ecosistemas, están enmarcadas dentro de los parámetros de la administración, manejo y apoyo para actuar en cualquier momento.

Estos lineamientos orientadores que se proponen se hacen tomando en consideración que estos ecosistemas a lo largo del tiempo han sido afectados y hasta devastados por diversos factores de orden social y físico-natural, destacándose entre ellos como los primordiales la planificación y las técnicas de manejo inadecuadas, así como las políticas de desarrollo sectorial inconsistentes y desarticuladas.

Siguiendo con el basamento conceptual, se toman en cuenta las consecuencias de un desarrollo urbano sin previsión frente al sistema ambiental, que se denota en que los sistemas físico-funcionales, de movilidad y de espacio público no presentan condiciones aptas, lo que ha generado deterioro a nivel urbano, degradación de calidad de vida, inseguridad, y con esto, la consolidación de dinámicas y una imagen de paisaje negativa para los habitantes. Lo planteado requiere generar una respuesta integral en la relación humedales y comunidad donde la parte beneficiada lo haga de manera racional; para ello es importante entender los conceptos que se plantean a continuación.

Cabe destacar el termino simbiosis, que se refiere al tipo de asociación que establecen dos individuos, sean animales o vegetales, donde al menos uno de ellos se beneficia. Es una relación permanente y estrecha entre dos organismos que llevan una vida común. Habitualmente al menos una de las especies obtiene un beneficio de dicha relación. Normalmente esta relación es específica entre dos especies concretas, ya sean animales, plantas, hongos o microorganismos (Contreras, 2014). Es evidente que existe una relación de simbiosis entre los humedales y las ciudades. Por este motivo, el desafío que se plantea actualmente es preservarlos para que las ciudades del futuro sean más prósperas y saludables.
Con respecto a la empatía existen múltiples definiciones, si bien la más aceptada y práctica es saber ponerse en el lugar del otro, poseer un sentimiento de participación afectiva en la realidad que afecta a otra persona. "La empatía es la intención de comprender los sentimientos y emociones, intentando experimentar de forma objetiva y racional lo que siente otro individuo" (Téllez, 2018, p.4).

Se destaca en la empatía cuando un individuo consigue sentir el dolor o el sufrimiento de los demás poniéndose en su lugar, despierta el deseo de ayudar y actuar siguiendo los principios morales. Esta capacidad de ponerse en el lugar del otro ayuda a comprender mejor el comportamiento en determinadas situaciones y la forma como el otro toma las decisiones. Para el caso de los humedales urbanos, la valoración de estos, para su manejo, gestión y políticas, y la combinación de instrumentos económicos y sociales, contribuye a una toma de decisiones equilibrada e incrementa la empatía de la población aledaña respecto a las decisiones que se tomen. Por lo tanto, conceptualizar el término simbioempatía está relacionado con la reciprocidad estrecha y permanente entre los seres vivos que ocupan un lugar en común, cuya participación es afectiva sin que se perturben entre sí.

Los humedales urbanos son los humedales que se encuentran dentro de los límites de ciudades, poblaciones y otras conurbaciones (Ramsar, 2016). Estos humedales de entornos urbanos ya están degradados o están degradándose debido al avance de las poblaciones de los alrededores, por la contaminación, el manejo inadecuado de los residuos y el relleno u otros fenómenos, sumado al hecho de que esas actividades han disminuido los servicios que el ecosistema puede prestar, lo que ha provocado el poco reconocimiento de su valor e importancia por parte de los órganos competentes y las comunidades urbanas.

Entre tanto, el urbanismo sostenible es el que produce menos transformación del ecosistema en el que se implanta; es decir, el que causa menor impacto posible sobre el ambiente, dirigido a consumir la menor cantidad de recursos y energía y generar la menor cantidad posible de residuos y emisiones, lo que se traduce en una mejor calidad de vida para generaciones futuras y un equilibrio dinámico entre la población, la ciudad y la naturaleza (Álvarez et al., 2014).

Se debe considerar que la ciudad es un ambiente artificial distinto de los ecosistemas 
naturales y sus dinámicas. Por lo tanto, no es un fin de la ciudad regresar a la vida silvestre, más bien se entiende como prioritaria la necesidad de propiciar el desarrollo de ecosistemas urbanos emergentes, consecuencia de la simbiosis entre el espacio físico y el espacio fundado en lo urbano y lo rural disminuyendo los costos ambientales y sin comprometer la capacidad de carga del espacio territorial.

\section{CASO VENEZOLANO}

Luego de conocer las concepciones que fundamentan los humedales urbanos y su problemática, es preciso tocar un caso específico donde se presenta una gran cantidad de ecosistemas con una riqueza extraordinaria de especies y una gran gama de legislación ambiental, que sin embargo adolece de buen manejo ambiental de su territorio. El lector se preguntará ¿por qué Venezuela? Porque ese país presenta ventajas comparativas referidas a su ubicación geográfica y a la riqueza natural de su territorio; así como, a nivel de ecosistemas, se destaca por su condición de ser Amazónico, Andino, Atlántico, Caribeño y Llanero, lo que hace de este país una real expresión del neotrópico. Posee una gran diversidad de humedales que contienen lagunas parameras y de montaña, ríos, manantiales, planicies de inundación, bancos de plantas acuáticas, ecosistemas anegados en los llanos, lagos y lagunas costeras, albuferas, arrecifes coralinos, manglares, ciénagas y morichales, entre otros. Además, posee una gran variedad de humedales artificiales, donde se incluyen tanques de reservas para el ganado y la acuicultura, tierras agrícolas periódicamente inundadas, salinas, plantas de tratamiento, represas y embalses, entre otros.

Venezuela es parte de la Convención Ramsar desde el año 1988, cuando designó al Refugio de Fauna de Cuare como área Ramsar. Posteriormente, en el año 1996 incorporó cuatro nuevas áreas a la lista de humedales de importancia internacional, siendo estos: el Refugio de Fauna Silvestre y Reserva de Pesca de Los Olivitos, el Parque Nacional Archipiélago de los Roques, el Parque Nacional Laguna de la Restinga y el Parque Nacional Laguna de Tacarigua, los cuales cuentan con una gran riqueza en cuanto a biodiversidad (especies de plantas y animales), e incluso protegen especies en peligro de extinción como las tortugas marinas y el caimán de la costa, entre otros (Ayube, 2012).
Estas áreas suministran importantes recursos alimenticios para las comunidades locales y para el resto del país; en general, se encuentran afectados por la carencia de recursos económicos para un manejo apropiado, la pesca ilegal y la contaminación ocasionada por la basura arrojada, tanto por pobladores como por turistas. Así, vale plantear algunos lineamientos básicos para la gestión de humedales urbanos, según Ramsar, referidos a:

1. Abordaje de la problemática del humedal en forma institucional concertada y holística mediante un sistema de gestión local.

2. Fortalecimiento del gobierno local desde el punto de vista institucional y de la readecuación de sus estructuras internas.

3. Implementación de estrategias de educación ambiental.

4. Ordenamiento urbano para la restauración y enriquecimiento de los cuerpos de agua.

5. Participación de todos los sectores involucrados.

6. La gestión ambiental debe ser una organización con atribuciones ejecutivas, que integre a la comunidad en la solución y control de los problemas ambientales, amparada en la legislación ambiental y un eficaz ejercicio del poder de policía municipal, haciendo frente a acciones agresivas que alteran la calidad ambiental, afectando los ecosistemas naturales en particular y las condiciones de vida de las comunidades en general, poniendo en riesgo a la sociedad. 


\section{S U S T E N T O S J UR Í D I C O S VENEZOLA NOS}

La base fundamental del ordenamiento jurídico ambiental de Venezuela se desprende de la Constitución de la República Bolivariana de Venezuela, CRBV (1999), en la que se incluye un capítulo sobre Derechos Ambientales, destacando los principios esenciales para la formulación e implementación de la política ambiental. De ella surge un cuerpo de leyes y normativas afines a la protección del ambiente, con énfasis en la conservación de los cuerpos de agua, las Ordenanzas Municipales.

En el ámbito internacional, en 1971 se patrocinó la Convención Ramsar, "Convención Relativa a los Humedales de Importancia Internacional, especialmente como Hábitat de Aves Acuáticas". También el Convenio sobre diversidad biológica de 1992, como marco de referencia para la generación y el desarrollo político y normativo con respecto a la conservación y protección de ecosistemas estratégicos En este último se busca la conservación de especies por medio de la adopción de medidas como la creación del Sistema de Áreas Protegidas y otros mecanismos especiales de protección.

En este caso en particular del humedal presente en el Jardín Botánico de San Carlos, se debería considerar un área de administración especial, el cual debe tener su base legal y legislación ambiental, pero carece de tal figura, la cual debe crearse para ser normado este espacio. Hasta ahora se desconoce su figura jurídica que permita crear una normativa de uso de sus espacios y evitar, de esta manera, los conflictos de uso presentes y los que se puedan generar.

Como se mencionó, la CRBV es el principal y más importante instrumento jurídico para la protección y uso racional de los humedales, documento que ampara los derechos fundamentales de los venezolanos y desde donde surge el derecho a un ambiente sano y ecológicamente equilibrado. En este sentido, la política nacional de los humedales se manifiesta en cumplimiento al mandato legal y político vigente en el país, partiendo de la CRBV (Gaceta Oficial $\mathrm{N}^{\circ} 5.453$ de fecha 24 de marzo, 2000), que declara en el artículo 127 que es de interés nacional la conservación y protección del ambiente en beneficio de sí misma y del mundo futuro en beneficio de la Nación.

Tal como lo cita el artículo 127 de la CRBV (2000): "Es un derecho y un deber de cada generación proteger y mantener el ambiente en beneficio de sí misma y del mundo futuro" (p.25). Así mismo dicta, "toda persona tiene derecho individual y colectivamente a disfrutar de una vida y de un ambiente sano, seguro y ecológicamente equilibrado". Además, establece: "El Estado protegerá el ambiente, la diversidad biológica, genética, los procesos ecológicos, los parques nacionales y monumentos naturales y demás áreas de especial importancia ecológica" (p.25). Por lo tanto, es una obligación fundamental del Estado, con la activa participación de la sociedad, garantizar que la población se desenvuelva en un ambiente libre de contaminación, en donde el aire, el agua, los suelos, las costas, el clima, la capa de ozono, las especies vivas, sean especialmente protegidas. En sí mismo, este artículo no especifica cuáles serán las acciones rectoras para proteger el ambiente, sino que enumera algunos elementos físicos amparándolos "de conformidad con la Ley". Esta Constitución ha sido considerada como un gran avance del derecho ambiental venezolano, puesto que contempla el instrumento de mayor jerarquía en la pirámide de las leyes el derecho de toda persona a un ambiente sano, legitimando a cualquier persona para reclamar el daño causado.

Entre tanto, el artículo 128 establece implementar una política de ordenación del territorio y su reglamento para el desarrollo de usos atendiendo a las realidades ecológicas, geográficas, poblacionales, sociales, culturales, económicas, políticas, de acuerdo con las premisas del desarrollo sustentable, que incluya la información, consulta y participación ciudadana, donde una ley orgánica desarrollará los principios y criterios para este ordenamiento (CRBV, G.O., 2000). A partir de esta carta magna se desprende un conjunto de leyes ambientales, pero la realidad es que, si bien no en todos los casos trata directamente el tema de los humedales, está íntimamente relacionada con los mismos, como por ejemplo las leyes que se aplican a la vida silvestre, al recurso forestal y legislación ambiental en general tratada a continuación.

\section{LEY ORGÁNICA DEL AMBIENTE $(2006)$}

Esta Ley estipula en su Artículo 10 lo siguiente: son objetivos de la gestión del ambiente, bajo la rectoría y coordinación de la Autoridad Nacional Ambiental: 8. Asegurar la conservación de un ambiente sano, seguro y ecológicamente equilibrado. 13. Resguardar, promover y fomentar áreas que coadyuven a la preservación de un ambiente seguro, sano y ecológicamente equilibrado. (p.13). 
Igualmente, el Artículo 34 invoca: la educación ambiental tiene por objeto promover, generar, desarrollar y consolidar en los ciudadanos y ciudadanas conocimientos, aptitudes y actitudes para contribuir con la transformación de la sociedad, que se reflejará en alternativas de solución a los problemas socio ambientales, contribuyendo así al logro del bienestar social, integrándose en la gestión del ambiente mediante la participación activa y protagónica, bajo la premisa del desarrollo sustentable (p.14).

Por su parte, el Artículo 48 dice: a los fines de la conservación de los ecosistemas, recursos naturales y de la diversidad biológica, serán objeto de medidas prioritarias de protección: 1 . Los ecosistemas frágiles, los de alta diversidad genética y ecológica y los que constituyan áreas de paisajes naturales de singular belleza o ecosistemas prístinos, poco intervenidos y lugares con presencia de especies endémicas y aquellos que constituyen hábitat y tierras de pueblos indígenas susceptibles de ser afectados en su integridad cultural. 2. Las especies o poblaciones de animales y plantas particularmente vulnerables, endémicas o que se encuentren amenazadas o en peligro de extinción. 3. Las especies raras o poblaciones de singular valor ecológico, científico, estratégico o económico, de utilidad actual o potencial. 6. Las áreas naturales que tengan un interés especial para su conservación. (p.15).

Asimismo, el Artículo 57 sostiene que: para la conservación de la calidad del agua se tomarán en consideración los siguientes aspectos: 1 . La clasificación de las aguas atendiendo a las características requeridas para los diferentes usos a que deba destinarse. 2. Las actividades capaces de degradar las fuentes de aguas naturales, los recorridos de estas y su represamiento. 6 . El seguimiento continuo y de largo plazo de la calidad de los cuerpos de agua (p.16).

Este instrumento jurídico que rige la gestión ambiental responde a la necesidad de la protección de los recursos naturales en beneficio de la colectividad para alcanzar el máximo bienestar de la población, y tiene por objeto establecer las disposiciones y los principios rectores para la gestión del ambiente, en el marco del desarrollo sustentable como derecho y deber fundamental del Estado y de la sociedad, para contribuir a la seguridad y al logro del máximo bienestar de la población y al mantenimiento del planeta, en interés de la humanidad.

\section{LEY PENAL DEL AMBIENTE (2012)}

La presente ley, en su Artículo 69, plantea lo siguiente: Destrucción de vegetación en las vertientes. El que deforeste, tale, roce o destruya vegetación donde existan vertientes que provean de agua las poblaciones, aunque aquella pertenezca a particulares, será penado con prisión de uno (1) a cinco (5) años y multa de mil (1.000) a cinco mil (5.000) unidades tributarias (p.16).

Mientras que el Artículo 84 dice: Vertido ilícito. El que vierta o arroje materiales no biodegradables, sustancias, agentes biológicos o bioquímicos, efluentes o aguas residuales no tratadas según las disposiciones técnicas dictadas por el Ejecutivo Nacional, objetos o desechos de cualquier naturaleza en los cuerpos de las aguas, sus riberas, cauces, cuencas, mantos acuíferos, lagos, lagunas o demás depósitos de agua, incluyendo los sistemas de abastecimiento de aguas, capaces de degradarlas, envenenarlas o contaminarlas, será sancionado con prisión de uno a dos años o multa de un mil unidades tributarias $(1.000$ U.T.) a dos mil unidades tributarias $(2.000$ U.T.) (p.19).

El Artículo 99 refiere: Disposición indebida de residuos o desechos sólidos no peligrosos. La persona natural o jurídica que infiltre o entierre en los suelos o subsuelos, sustancias, productos o materiales no biodegradables, agentes biológicos o bioquímicos, agroquímicos, residuos o desechos sólidos o de cualquier naturaleza que no sean peligrosos, en contravención a las normas técnicas que rigen la materia, que sean capaces de degradarlos, esterilizarlos, envenenarlos o alterarlos nocivamente, será sancionada con arresto de uno a tres años o multa de trescientas unidades tributarias $(300$ U.T.) a un mil unidades tributarias (1000 U.T.) (p.21).

En lo expuesto se refleja las sanciones a las que están sometidos los que infrinjan la Ley orgánica del ambiente en cuanto al uso indebido de los recursos naturales, buscando asegurar la vida útil de los ecosistemas frágiles, en este caso los humedales.

\section{LEY DE LA DIVERSIDAD BIOLÓGIGA (2000)}

El Artículo 3 de la presente ley expresa: El patrimonio ambiental de la Nación lo con- 
forman los ecosistemas, especies y recursos genéticos que se encuentren dentro del territorio nacional y su ámbito jurisdiccional, incluyendo la zona marítima contigua y la zona económica exclusiva. (p.1).

En tanto que el Artículo 4 dice: A los efectos de esta Ley, la conservación de la diversidad biológica comprenderá fundamentalmente de: la conservación y la regulación del manejo, in situ y ex situ, de la diversidad biológica. 3. La promoción de la investigación y la capacitación de los recursos humanos, para un adecuado conocimiento de la diversidad biológica. 15 La promoción de educación ambiental y la divulgación para incentivar la participación ciudadana con relación a la conservación y uso sustentable de la diversidad biológica. 17. El reconocimiento y la preservación del conocimiento que sobre la diversidad biológica y sus usos tienen las comunidades locales. En este sentido, los humedales son ecosistemas extremadamente ricos en especies de plantas y animales que, por estar ubicados en zonas de transición entre ambientes acuáticos y terrestres, son zonas de alta productividad, pues son lugares de desarrollo de infinidad de especies de agua dulce, lo que hace que esta sea una de las leyes que tiene una relación directa con este tipo de ecosistemas (pp.1,2).

\section{LEY DE BOSQUES (2013)}

Esta Ley, en su Artículo 5 enuncia: Utilidad pública e interés social. Se declaran de utilidad pública e interés social: 1. Los bosques nativos localizados en todo el territorio nacional, representados por los ecosistemas boscosos primarios que se han formado y desarrollado naturalmente con las especies forestales autóctonas de las distintas regiones del país, cuya cobertura arbórea sea mayor o igual al diez por ciento $(10 \%)$, y abarquen una superficie mínima de mil metros cuadrados.

De igual manera, el Artículo 6 se refiere a: Interés público. Se declara de interés público el ejercicio de las competencias o atribuciones de los órganos o entes del poder público relacionadas con: a.- Preservación de especies y bosques nativos de especial valor ecológico. b.- Fomento de bosques en todo el territorio nacional. c.- Promoción y difusión de los valores de los bosques venezolanos. d.- Inclusión y participación de la ciudadanía en la gestión de los bosques. e.- Investigación y nuevas tecnologías para el desarrollo forestal sustentable. f.- Prevención y control de ilícitos contra el patrimonio forestal. g.- Fortalecimiento de la cadena productiva forestal (p.2).

Bien interesante el Artículo 12 que corresponde a los Municipios, en el ámbito de sus competencias: 5. La conservación y resguardo de los bosques nativos ubicados en ejidos municipales. 6. El fomento y promoción de actividades orientadas a la consolidación de la cadena productiva forestal local. 7. El apoyo a los programas nacionales de formación y difusión de la cultura del bosque (p.3).

Como su nombre lo indica, esta ley tiene una marcada disposición protectora de bosques, áreas forestales en general y zonas protectoras como figuras de áreas bajo régimen de administración especial, por lo cual su relación con los humedales es inminente, debido a que estos ecosistemas están caracterizados por la presencia de bosques de diferentes magnitudes.

\section{LEY DE AGUAS (2007)}

La presente Ley en su Artículo 4 habla de: La gestión integral de las aguas tiene como principal objetivo:

1. Garantizar la conservación, con énfasis en la protección, aprovechamiento sustentable y recuperación de las aguas, tanto superficiales como subterráneas, a fin de satisfacer las necesidades humanas, ecológicas y la demanda generada por los procesos productivos del país (p.3).

En su Artículo 5 destaca: Los principios que rigen la gestión integral de las aguas se enmarcan en el reconocimiento y ratificación de la soberanía plena que ejerce la República sobre las aguas y son: 7. Los usuarios o usuarias de las aguas contribuirán solidariamente con la conservación de la cuenca, para garantizar en el tiempo la cantidad y calidad de las aguas. 8. Es una obligación fundamental del Estado, con la activa participación de la sociedad, garantizar la conservación de las fuentes de aguas, tanto superficiales como subterráneas (p.3).

Como se ha señalado, los humedales engloban una gran cantidad de ambientes y comparten una propiedad diferenciándola de los ecosistemas terrestres es la presencia de agua, siendo un recurso fundamental en la determinación de sus funciones ecológicas, por lo tanto, esta ley es esencial para regir estos ecosistemas. 


\section{LEY ORGÁNICA DE ORDENAGIÓN DE TERRITORIO (1983)}

A los efectos de esta Ley, en su Artículo 2 dice: Se entiende por ordenación del territorio la regulación y promoción de la localización de los asentamientos humanos, de las actividades económicas y sociales de la población, así como el desarrollo físico espacial, con el fin de lograr una armonía entre el mayor bienestar de la población, la optimización de la explotación y uso de los recursos naturales y la protección y valorización del medio ambiente, como objetivos fundamentales del desarrollo integral.

Igualmente, el Artículo 3 reza: A los efectos de la presente Ley orgánica, la ordenación del territorio comprende: 1 . La definición de los mejores usos de los espacios de acuerdo con sus capacidades, condiciones específicas y limitaciones ecológicas. 2. El establecimiento de criterios prospectivos y de los principios que orienten los procesos de urbanización, industrialización, desconcentración económica y de asentamientos humanos. 9. La protección del ambiente, y la conservación y racional aprovechamiento de las aguas, los suelos, el subsuelo, los recursos forestales y demás recursos naturales renovables y no renovables en función de la ordenación del territorio (p.1). Mientras que el Artículo 5 destaca que son instrumentos básicos de la ordenación del territorio, el Plan Nacional de Ordenación del Territorio, y los siguientes planes en los cuales este se desagrega: d. Los planes de las áreas bajo régimen de administración especial (p.2).

En definitiva, puede observarse la importancia que reviste el contenido de esta Ley, por cuanto garantiza la continuación de los distintos planes de ordenación en beneficio del aprovechamiento sustentable de los recursos naturales. Sin embargo, aunque esta ley no tiene relación directa con los humedales, existe de manera directa su vinculación, puesto que en estos ecosistemas se localizan asentamientos humanos que hacen uso de los recursos naturales y desarrollan actividades económicas y sociales; en tal sentido, son estas poblaciones las mediadores de que se logre una armonía entre el mayor bienestar de la población, la optimización de la explotación y uso de los recursos naturales y la protección y valorización del ambiente, como objetivos fundamentales del desarrollo integral.

\section{LEY DE GONSEJOS GOMUNALES (2009)}

El Artículo 28 de esta Ley dice: La Asamblea de Ciudadanos y Ciudadanas elige el número de voceros o voceras postulados de acuerdo con la cantidad de comités de trabajo u otras organizaciones comunitarias que existan o se conformen en la comunidad, tales como: 9. Comité de mesa de agua, y a. Comités de ambiente y demarcación de tierra en el hábitat indígena. (No establece su existencia en otros territorios) (p.9).

Esta Ley se relaciona con la investigación, debido a que favorece la organización de las comunidades para tratar, entre otros, el tema ambiental con la conformación de los comités de ambiente, los cuales tienen como función la solución de la problemática ambiental presente, mediante la elaboración de proyectos que respondan a resolver dicha problemática, siendo una gran herramienta para la conservación de los humedales urbanos.

\section{LEY DE PROTEGGIÓN DE FAUNA SILVESTRE (1970)}

En el Artículo 5 de la presente Ley destaca que: Se declara de utilidad pública: 1.- La creación de reservas, refugios y santuarios de fauna silvestre. 2.- La conservación, el fomento y aprovechamiento racional de la fauna silvestre. 5.- La conservación y fomento de los recursos que sirvan de alimentación y abrigo a la fauna silvestre. 6.- La investigación científica de la fauna silvestre (p.1).

Así mismo, el Artículo 14 plantea: El Estado tiene la obligación de realizar y fomentar la investigación científica conducente a la utilización racional de la fauna silvestre y establecerá los centros de investigación que fuesen necesarios. A este fin, los propietarios deberán permitir la entrada de los funcionarios competentes a sus respectivos fundos, con el objeto de que ellos puedan colectar animales vivos o muertos y realizar cualquier otra actividad que se requiera para dichas investigaciones (p.2).

En tanto que el Artículo 18 dice: El Ejecutivo nacional tomará las medidas necesarias para preservar, modificar o restaurar el hábitat de los animales silvestres (suelos, flora, aguas), que requieran los planes de ordenación y manejo, y adoptará las resoluciones que estime convenientes para evitar contaminaciones de cualquier naturaleza que pudieran afectar el 
hábitat de la fauna silvestre; igualmente procurarán en lo posible sanear aquellos ambientes que hayan sido afectados. (p.2).

En su Artículo 31 señala: El Ejecutivo nacional declarará refugios de fauna silvestre aquellas zonas del territorio nacional que, previo el estudio científico correspondiente, se estimen necesarias para la protección, conservación y propagación de animales silvestres, principalmente de aquellas especies que se consideren en peligro de extinción, ya sean residentes o migratorias. (p.3). Con lo expuesto, se debe destacar que en las áreas Ramsar de Venezuela se encuentran dos refugios de fauna considerados: el refugio de fauna silvestre de Cuare y el refugio de fauna silvestre y reserva de pesca Ciénaga de los Olivitos, ambas figuras se encuentran con planes de ordenamiento y reglamentos de uso que las rige.

\section{LEY ORGÁNICA DE TURISMO (2008)}

Se destaca el Artículo 6, puesto que indica que: del Sistema Turístico Nacional, a los efectos de este decreto con rango, valor y fuerza de Ley orgánica de turismo, se entiende por Sistema Turístico Nacional el conjunto de sectores, instituciones y personas, quienes relacionados entre sí contribuyen al desarrollo sustentable de la actividad turística, bajo los principios de cooperación, coordinación e información interinstitucional, sustentabilidad ambiental, integridad territorial, corresponsabilidad y solidaridad (p.1).

\section{LEY DE GESTIÓN DE LA DIVERSIDAD BIOLÓGIGA. GACETA OFICIAL 5468 DE 24.05 .2000}

El trascendental del Artículo 2 es que: A los efectos de la presente Ley, la gestión de la diversidad biológica comprende: 1 . La conservación de todos sus componentes a fin de preservar o restablecer los equilibrios ecológicos que permitan al hombre y demás seres vivos convivir en armonía con su ambiente. 2. El manejo y aprovechamiento de los diferentes componentes de la diversidad biológica, bajo principios de sustentabilidad ecológicos y bioéticos, respetando los valores culturales y considerando la participación justa y equitativa de la población en los beneficios que se deriven de aquellos. 3. La investigación y la generación de conocimientos sobre la diversidad biológica, a los fines de su conservación, aprovechamiento sustentable y manejo. (pp. 2 y 3 ).

Así mismo comprende el punto 6 la compatibilización entre las actividades antrópicas y el mantenimiento de los bienes y servicios ambientales que se deriven de la diversidad biológica. 7. La prevención, seguimiento, evaluación, mitigación, corrección y reparación de las alteraciones a la diversidad biológica causadas, directa o indirectamente, por actividades humanas o eventos naturales extremos. 8. La declaratoria de áreas bajo régimen de administración especial con la finalidad de proteger y manejar in situ los diversos componentes de la diversidad biológica y los procesos ecológicos inherentes. 9. La adopción de acciones y medidas para la vigilancia y el control de actividades capaces de degradar la diversidad biológica y modificar negativamente los procesos ecológicos propios de ella.

De igual manera, indica: 15. La educación ambiental como un proceso continuo, interactivo e integrador, orientado a crear conciencia en los ciudadanos, ciudadanas y comunidad en general, para la participación protagónica en la gestión compartida de la diversidad biológica. 17. La promoción y divulgación de la información y el conocimiento, sobre todos los aspectos relacionados con la gestión de la diversidad biológica, a través de los medios de comunicación social (p.3).

Mientras que en el Artículo 69 dice: la Autoridad Nacional Ambiental, en la implementación de la Estrategia nacional de la diversidad biológica, establecerá el sistema nacional de conservación in situ con base en las bio-regiones que conforman el territorio nacional, los ecosistemas de importancia estratégica, de prioridad de protección y el Sistema Nacional de Áreas bajo régimen de administración especial (p.12).

También el Artículo 85 indica: la Autoridad Nacional Ambiental establecerá limitaciones o prohibiciones al aprovechamiento de los componentes de la diversidad biológica, con énfasis en los casos siguientes: 1. Cuando se trate de especies endémicas, raras, vulnerables, amenazadas o en peligro de extinción. 2. Cuando se trate de ecosistemas en condiciones de vulnerabilidad o fragilidad o hayan sido declarados de importancia estratégica. 3. Cuando amenace la salud humana o los elementos esenciales de la identidad cultural de los pueblos. 4. Cuando pueda generar impactos ambientales negativos o difícilmente controlables. 5. Cuando exista el riesgo de pérdida de la diversidad biológica y sus 
componentes. 6. Cuando existan restricciones específicas que deriven de normas especiales sobre bioseguridad. 7. Cuando se trate de áreas bajo régimen de administración especial (p.14).

En materia de gestión de la diversidad biológica, la formulación y ejecución de la política, deberá incorporarse la prevención de los daños a sus componentes y entorno para promover procesos participativos de análisis y resolución de conflictos. De manera tal que, de acuerdo con el principio de conservación de los ecosistemas, el país debe llevar a cabo acuerdos o convenios internacionales, en cuanto a la prevención y tratamiento de los daños causados a los componentes de la diversidad biológica; esta última de gran magnitud en los humedales urbanos.

Se debe recalcar que en esta Ley se plantea para la conservación de la diversidad biológica la educación ambiental y la participación ciudadana, entre otras, como vías para acceder a la investigación y conocimiento de la diversidad biológica.

\section{O N V E I O S}

\section{N T E R N A G I O N A L E S}

En primer lugar, se destaca la Convención Relativa a los Humedales de Importancia Internacional, especialmente como hábitat de aves acuáticas, Ramsar (1971). Según esta Convención, los humedales son pantanos de régimen natural o artificial, permanente o temporal, estancado o corriente, dulce o salobre, que no excedan de 6 metros de profundidad. Precisamente, una de las causas principales para la convocatoria inicial del convenio de Ramsar (Irán), fue la constancia de la disminución del número de aves acuáticas y la desecación en años anteriores de numerosas zonas húmedas, debido a la intervención antrópica; en el caso de los humedales urbanos, la causa principal es el incremento del proceso de urbanismo. Se trata de un acuerdo internacional firmado en la ciudad iraní de Ramsar, en 1971, cuyo objetivo es la conservación y el uso racional de los humedales mediante acciones locales, regionales y nacionales, y gracias a la cooperación internacional, como contribución al logro de un desarrollo sustentable en todo el mundo.

Otro convenio de gran importancia es el Convenio Marco sobre la Diversidad Biológica (1992), el cual, en su Artículo 1 plantea: Los objetivos del presente Convenio, que se han de perseguir de conformidad con sus disposiciones pertinentes, son la conservación de la diversidad biológica, la utilización sostenible de sus componentes y la participación justa y equitativa en los beneficios que se deriven de la utilización de los recursos genéticos, mediante, entre otras cosas, un acceso adecuado a esos recursos y una transferencia apropiada de las tecnologías pertinentes, teniendo en cuenta todos los derechos sobre esos recursos y a esas tecnologías, así como mediante una financiación apropiada.

En su Artículo 2: A los efectos del presente Convenio: Por "área protegida" se entiende un área definida geográficamente que haya sido designada o regulada y administrada a fin de alcanzar objetivos específicos de conservación (p.1).

Con este convenio, se reitera el objetivo de mantener y restaurar los humedales, sus recursos y biodiversidad, para las generaciones futuras. Igualmente, se busca que los humedales y los recursos hídricos se conservan y se gestionen teniendo en cuenta toda la escala de sus valores y servicios, para beneficio de la biodiversidad y del bienestar humano en el apoyo que prestan los humedales a los sistemas de sustento. Esto refleja un equilibrio entre las necesidades habituales y las emergentes en el área de la conservación y manejo de los humedales. Por lo tanto, es indispensable que se tomen en cuenta los esfuerzos internacionales que se buscan con estos convenios y se apoyen la conservación y uso racional de los humedales dentro del ámbito del desarrollo sustentable.

En este sentido, la conservación de los humedales es vital para alcanzar los objetivos de preservación de la biodiversidad previstos en los tratados internacionales y las obligaciones conexas que se derivan de ellos. Los humedales desempeñan una función importante en el logro de estos objetivos, como:

-Mantenimiento de los procesos ecológicos esenciales y los sistemas que sustentan la vida: los humedales desempeñan estas funciones de diversas maneras; algunos mantienen y mejoran la calidad del agua, algunos regulan los caudales y reducen las inundaciones y pueden incrementar el caudal de los cursos de agua durante el verano; y algunos recargan los reservorios de aguas subterráneas. Los humedales son importantes como lugares de reproducción y descanso de aves acuáticas migratorias, como zonas de desove y cría de peces, y como hábitat de muchos invertebrados, reptiles, anfibios y plantas. 
-Preservación de la diversidad genética: los humedales desempeñan una función esencial en el mantenimiento de poblaciones de especies silvestres al servir de hábitat a una fauna y flora variadas. Cerca de un tercio de las especies silvestres amenazadas, en peligro o raras identificadas viven en los humedales.

-Aprovechamiento sostenible de especies y ecosistemas: muchas economías locales y provinciales o estatales y territoriales dependen directamente de los recursos de los humedales, como peces y especies silvestres, productos de origen vegetal y madera.

De igual forma, el Convenio de Estocolmo sobre contaminantes orgánicos persistentes, vigente 17.05.2004, que establece en su Artículo 1: Teniendo presente el criterio de precaución consagrado en el principio 15 de la Declaración de Río sobre el Medio Ambiente y el Desarrollo, el objetivo del presente Convenio es proteger la salud humana y el medio ambiente frente a los contaminantes orgánicos persistentes. Este Convenio reconoce que los contaminantes orgánicos persistentes tienen propiedades tóxicas, son resistentes a la degradación, se acumulan y son transportados por el aire, el agua y las especies migratorias por medio de las fronteras internacionales y depositados lejos del lugar de su liberación, acumulándose en ecosistemas terrestres y acuáticos. Al mencionar estos ecosistemas, se incorporan los humedales como tales (pp.1 y 2).

Vale destacar que, a pesar de que Venezuela posea una rica y variada base legal propia y a nivel internacional que ampara el referido tema, no se le dé la importancia en cuanto al manejo de los humedales y aquí entra el interés político para ejecutar su conservación. No hay que dejar de lado que en un país tan convulsionado producto de los problemas sociales, económicos y políticos se hace la tarea más difícil, aunado al empeño de los entes gubernamentales de explotar sus recursos naturales sin ningún tipo de control, administración ni medida; y lo que empeora la situación es la ausencia de la voz por parte de organismos internacionales que frenen tal actitud hacia el medio natural. Es imperativo que esta situación se revierta y se genere con premura la simbioempatía como eje de articulación hacia una responsabilidad social compartida hombre-naturaleza, no solo en el tema de humedales urbanos, sino en todo lo relacionado con la conservación del entorno natural.

Siguiendo con el contexto teórico que enriquezcan lo expresado, se debe tener claro los conceptos de economía digital y las ciudades inteligentes (smart cities), así como responsabilidad social. Con respecto al primero, muchos teóricos la definen como la vinculación de la ciencia y la tecnología con las empresas de producción y la sociedad. Es un un proceso complejo que implica cambios en la organización social, económica y política de los países, por lo tanto, es un facilitador para el desarrollo, puesto que se presenta como una nueva forma de producción y consumo. Otros sostienen que la economía digital se compone como un ecosistema, en el que se interrelacionan la infraestructura de las comunicaciones, los servicios y las tecnologías, y los usuarios finales, lo que finalmente determinará el nivel de desarrollo y avance de cada país. Así mismo, son variadas las posiciones y críticas de científicos, muchos con visiones radicales en cuanto a su relación con el ambiente, pero más adelante se profundizará en el tema. Lo planteado se puede evidenciar esquemáticamente en la figura siguiente.

De igual forma, se hará con lo concerniente a las ciudades inteligentes, pero se debe tener alguna noción de su significado. Estas han sido definidas como las ciudades caracterizadas por el manejo de la tecnología e innovación con el fin de hacer un mejor uso de los recursos, fomentando el desarrollo sostenible con miras a mejorar la calidad de vida de sus habitantes. Posteriormente, se mencionará su afectación sobre el ambiente natural. En general, todo este engranaje de conceptos y criterios conllevan a un compromiso de generar simbioempatía en los humedales urbanos como eje de articulación hacia una responsabilidad social compartida entre la sociedad y su entorno natural. 
F I G U R A 3 .

Concepciones en humedales urbanos

Fuente: elaboración propia (2021).

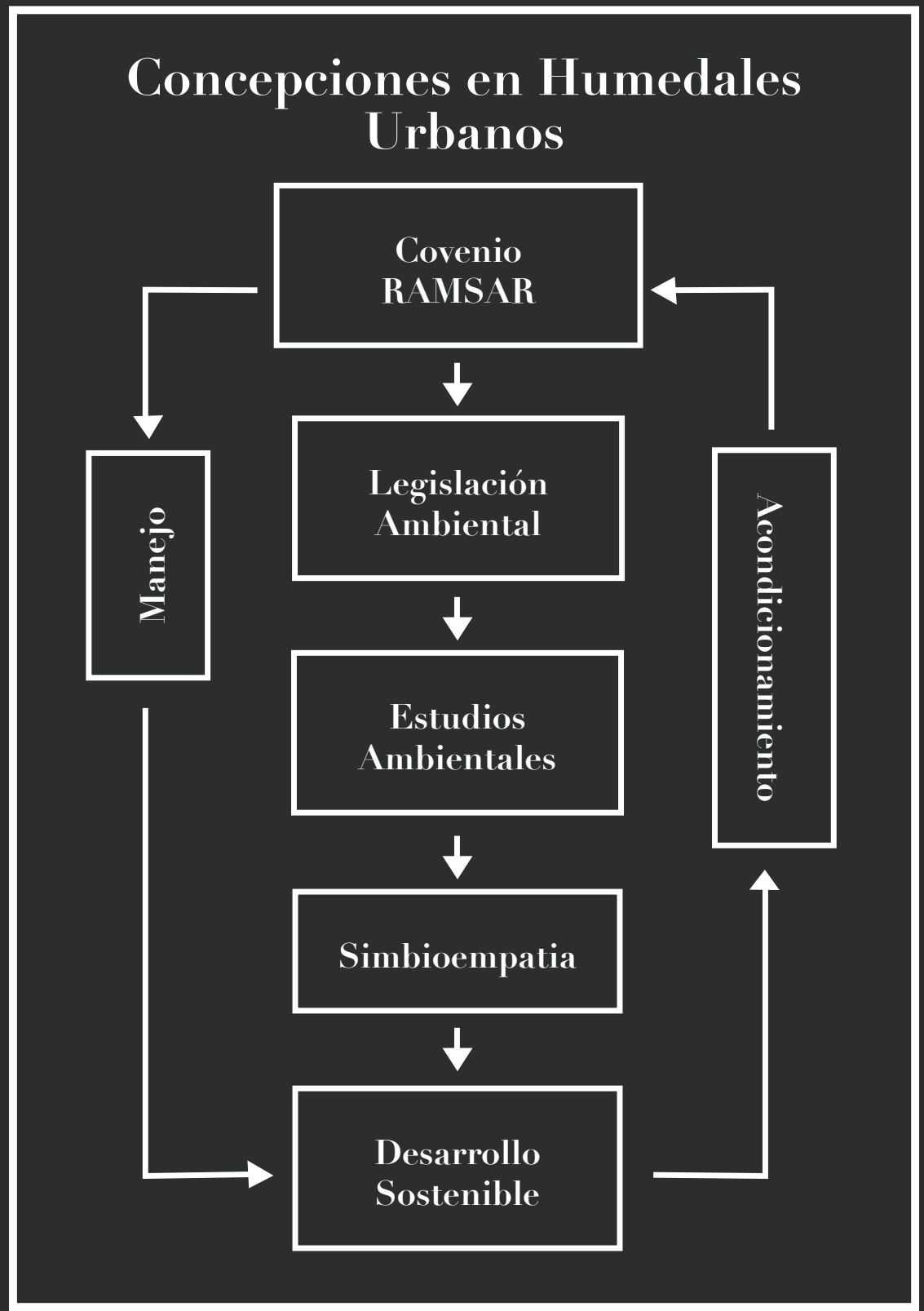

